\title{
Integrating complexity theory with health social work practice when working with senior people
}

\author{
Shurui Wang ${ }^{1 *}$, Yuanyuan Wang ${ }^{2}$ \\ ${ }^{1}$ School of Social Sciences, University of Tasmania, Launceston, Australia \\ ${ }^{2}$ College of Continuing Education, Beijing Institute of Technology, Zhuhai, China
}

\author{
Keywords \\ Health social work practice \\ Senior people \\ Complexity theory \\ CAS
}

Received: 2 April 2019 Accepted: 6 May 2019 Published: 28 June 2019

\begin{abstract}
Social work intervention in the contemporary healthcare system has proved a challenging practice for health workers, especially when working with senior people whose service experience always varies following nonlinear dynamics rather than linear dynamics. As social work services provided in hospitals are always perceived as the practice in "Complex Adaptive Systems (CAS), the complexity theory, which reflects continually changing needs and environments, can provide a new pathway for hospital social workers to manage the above-mentioned cases. Herein, the aim of this research is to address the feasibility and adaptability of implementing the complexity theory in hospital social work practice with a particular focus on senior service users. Firstly, this study will analyse the factors that influence the effects of service delivery for those seniors in hospitals and explain why these cases often are perceived as complex. Then it will introduce the complexity theory and CAS with the explanation of some related basic phrases such as non-linearity, embeddedness, and attractor patterns. In the next section, how the complexity theory can be embedded in hospital social work practice and guides social workers to reform their framework for practice will be addressed. The complexity theory will be used as a complementary theory to support social work practitioners to build a multidimensional understanding of complex cases and, in addition, to impact their practice. At last, the conclusion that the complexity theory will positively influence social work practice when dealing with complex cases related seniors will be drawn as the end of this paper.
\end{abstract}

(C) 2019 The Author(s). Published by TAF Publishing

\section{INTRODUCTION}

Social work practice always has to be conducted in a complex context constrained by multi-level forces which include macro forces like neoliberalism and managerialism, and micro forces like family supports and cooperation degree between social workers and clients. For social work services provided in hospitals, the impacts of these constraint conditions increase because of the freeze or contraction of state and federal health funding and the need to cooperate with other professionals in a multidisciplinary team. In fact, hospital social workers are often required to do more with less and need to define the distinctive elements of their role in the allied health department, which increase their working difficulties when dealing with complex cases. It is reported in (Australian Institute of Health and Welfare,
2018), about one-fifth Emergency Department (ED) presentations were people aged 65 and over in 2016-2017, and these people made over 4 times claims for specialists services compared with their counterparts who are under 65 (Australian Department of Human Services, 2017). Because influence factors for seniors' health are impacted by the interactions among multiple-layered CAS such as the illness or disease itself, patients' emotion, family supports and the hospital resources (Campbell et al., 2007; Dong \& Keshavjee, 2016; Newhouse \& Spring, 2010), many social workers in the hospital, especially those practitioners with less experience, describe their work experience with aged patients as stressful, full of anxiety, and sometimes powerless (McAlinden, McDermott, \& Morris, 2013; Yi \& Chia, 2018). Thereby, methods that can help hospital social workers de-

${ }^{*}$ corresponding author: Shurui Wang

†email: shurui.wang@outlook.com 
construct these complex cases and enhance the quality of service delivery should be raised up. Since the promotion of complexity theory, many pieces of research have been done to analyse the continually increased complexity in healthcare networks, and healthcare organizations including public hospitals are widely perceived as complex systems (P. Plsek, 2003; Israel \& Wolf-Branigin, 2011; Tokuda, 2016). Miller, Crabtree, McDaniel, and Stange (1998) put their focus on analysing the reasons why some interventions produce changes in primary care practice while others do not, and promoted joining, transforming and learning as three strategies that contribute to successful change. P. E. Plsek and Greenhalgh (2001) acknowledged the complex nature of healthcare systems and argued clinical practitioners should replace traditional "reduce and resolve" approaches with an intuitive view that accepts unpredictability, dynamic and creativity. Kannampallil, Schauer, Cohen, and Patel (2011) researched the complexity healthcare environments and adopted the degree of interrelatedness to indicate how different levels of complexity impact modern healthcare practice. However, most of these studies mainly focus on describing and analysing the contexts and characteristics of the healthcare systems, but rare of them provided applicable strategies that can ease the difficulties of real practice in hospitals.

As Fish and Hardy (2015) noted, the fleeting development of complexity theory provides an alternative approach to explore and analyse the 'non-linear' nature of many phenomena. The integration of this theory also has a profound effect on practice in healthcare organisations. Litaker, Tomolo, Liberatore, Stange, and Aron (2006) distinguished the complexity theory as mathematical complexity and aggregate complexity, and proposed the "STEP-UP" strategy to help primary care practitioners identify both successes and missed opportunities created by their current approach. While Tuffin (2016) studied the implications of complexity theory for healthcare systems and argued that meaningful changes are more likely to occur if the connections and interaction patterns between individual components can be understood properly.

However, even though the contents of complexity theory have been widely understood, in social work field, this theory is still mainly applied to understand complex phenomena at a conceptual level (Hudson, 2000; Warren, Franklin, \& Streeter, 1998; Woehle, 2007) and only a few pieces of research apply it to analyse the dynamic data patterns of those complex cases (Warren, Hawkins, \& Sprott, 2003). Therefore, in this paper, after addressing the social and individual problems that are commonly faced by senior people, com- plexity theory will be introduced as a theoretical paradigm to support social work practice in hospitals. It is shown that the complex theory can help social workers properly analyse and understand the interactional components related to decision-making, which further influences their framework for practice. As a result, through integrating the complexity theory with social work practice, the quality of aged care service in hospitals will be significantly improved.

\section{THE COMPLEX SITUATION FACED BY AGED-CARE SO- CIAL WORKERS IN HOSPITALS}

As an important component of professional social work practice, social work services provided in hospitals mainly include: screening and case finding, discharge planning and case management, counselling and emotional support, advocacy, referral and advice, and providing information related to decision-making to clients and their carers etc.. Among all the provided services, working with senior patients accounts for a large part in hospital social work practice because of the high admission and revisiting rate of aged people. This population also shows a high frequency of social service utilization, which further lead to overburdened caseload for social workers.

As is outlined by (Albert \& Davia, 2011), about half of hospital social workers reported their monthly caseloads were over 60 in 2010. However, since the hospital social work is defined as an essential intermediary occupation whose working scope is heavily dependent on other professions like medicine (Lymbery, 2006), many social work departments in the hospital are chronically under a situation of staff shortage. As a result, with the underestimated professional status, social workers in the hospital often have to face the dilemma of both keeping the balance between managing the heavy caseloads and providing qualified services to aged clients.

In addition, social, political, economic and cultural factors of the elders also increase difficulties for hospital social workers to work with frail elder patients. For instance, in a neoliberalism society, the activation of the Health Care (2010) and the continual budget cuts forced almost all hospitals to choose speedy discharges as their priority. Under this circumstance, the complexity of hospital social work also is dramatically increased because social workers have to balance the time demands of complex clients and the discharge requirements for hospitals. In fact, many social workers in the hospital describe their works as unpredictable and challenging and feel hard to provide suitable services to the clients. Some other influential contexts can be the socioeconomic status and socio-cultural factors of senior pa- 
tients. It is outlined that temperate personal habits and high social stratum often lead to a high-frequency utilization of healthcare, while socio-cultural factors have a significant impact on the way in which illness is interpreted, perceived and responded to (Omorogbe, 2018). All of these factors interact with each other and even one slight change could impact the effectiveness of service delivery. Social workers need to devote a significant amount of time to build a trust relationship with the service users so that they can logically analyse elder clients' strengths and weaknesses. Therefore, it is reasonable for social workers to perceive their works with aged people as complex since seniors' health condition are always deeply connected with their broad contexts.

The complex nature of seniors' health condition is viewed as another complex factor that constrains the quality of social work services provided to older clients. On the one hand, most seniors are suffered from some chronic diseases like dementia, disability and diabetes. All of these diseases can significantly impair their capability of decision-making and enhance their need for long-term and special care. Just as Redfern, Burton, Lonne, and Seiffert (2016) pointed out, the aged individual with a disability usually associates with serious medical conditions and life crisis, which call for significant care and support beyond their acute hospital treatment. On the other hand, social determinants of health such as income inequality, lower educational levels and poor working conditions in early life also constrain the health outcomes of senior service users. To be specific, it has been found that socially differentiated material resources and income inequality have an unequally effect on the distribution of health outcomes and health care of elderly (Craig, Bejan, \& Muskat, 2013). While lower educational levels, which are closely connected with individuals' health behaviours, income and literacy level, also have been proved as a significant factor that leads to adverse health outcomes like shorter life expectancies in later life (Albert \& Davia, 2011). Factors like poor working conditions in early life like occupational status, high level of work-life conflicts, workrelated stress and a lack of job control (McNamara, Bohle, \& Quinlan, 2011) have been proved to have a positive correlation with adverse health outcomes of senior people.

Besides the above-mentioned points, the differences of social support and family among aged patients also require social workers flexibly change their working procedures, which, on the other hand, increases the complexity of working with senior people. It is noted by Saito, Sagawa, and Kanagawa (2005) that social support can help senior people generate feelings like emotional closeness and a sense of belonging and has a positive effect on health status and well-being among elderly people. In the meanwhile, family members often are the primary caregivers of senior people and this intergenerational relationship often play a significant role in the family life of aged people due to the change of their family role from the giver to the receiver. With such connection, the inquiry of clients' social network and their families has become a necessary strategy for hospital social workers to find possible supports that can help the recovery of senior individuals. However, according to Hautsalo, Rantanen, and Astedt-Kurki (2013), the older population is a very heterogeneous group with variable needs and requirements. Therefore, it is impossible for social workers to summary a common method that can be adopted in all situations due to the uniqueness of each case related to aged people.

\section{COMPLEXITY THEORY AND CAS}

Complexity theory develops from the open-system view. It not only inherits the characteristic of eco-system theory that acknowledges all the components of a system are interconnected and interrelated, but also emerged as a new theoretical approach that can explain any kind of complex system. Just like eco-system theory, the complexity theory has been widely applied to many disciplines that are perceived as 'not simple', and has been used to solve many problems ranging from complicated to intractable since the beginning of the 21st century. As described by Woehle (2007), complexity theory is not a theory per se, but rather a loose collection of concepts, heuristics, and analytic tools that addresses fundamental questions on the nature of systems and their changes. In the healthcare field, the complexity theory has been adopted to improve care related interventions as it 'acknowledges the uniqueness of primary care practices and encourages flexibility in the form of intervention implementation, while maintaining fidelity to its essential functions' (Litaker et al., 2006). Since hospital social work practice has some similarities with primary care practice in nature, it is reasonable to integrate complexity theory with such professional to improve the understanding of complex cases among social workers.

In order to apply complexity theory to healthcare social work, the concept of CAS can be utilized to strengthen the understanding of individual components and provide better clarity on the holistic nature of the system. It is described by Litaker et al. (2006), P. E. Plsek and Greenhalgh (2001), P. Plsek (2003) that a CAS is a collection of individual agents that can act in flexible ways that are not always predictable but interconnected as the actions of one agent change the contexts of other agents. Some common exam- 
ples of CAS include the immune system, the financial market and collection of humans like families. For identifying the nature of the system, social workers and researchers can distinguish the inherent characteristics of the integrated system and components in terms of interrelations and predictability. The complexity of the system can be determined by using the coordinate shown in Figure 1.

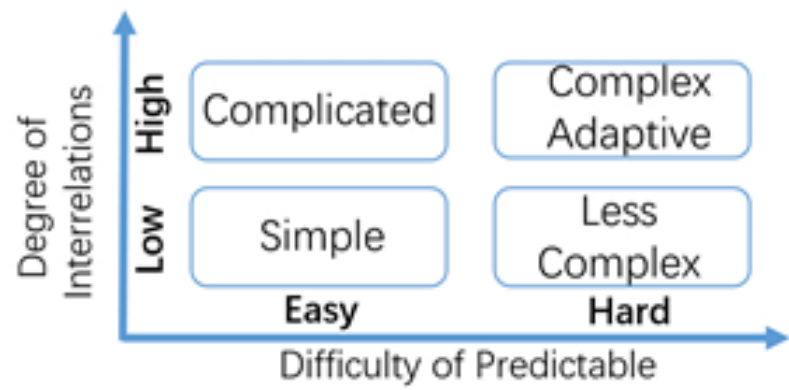

FIGURE 1. The coordinate for distinguishing the system as a CAS

It is shown that, in comparison with the simple system, the complex adaptive system is characterized a the high degree of interrelationship. Such complexity is coupling with the high computational expensive in predicting the system's output. This implies that the components of the system present a high degree of interrelatedness, which increased the challenge to predict the performance of the system before making decisions.

Underneath this frame, the key features of the CAS are generally shown in the aspects of (1) Predictability: embeddedness, non-linearity, and co-evolution; (2) Interrelation: Selforganization and attractor patterns etc., (Long, McDermott, \& Meadows, 2018). Specifically, the characteristics of a CAS can be described as follows:

- Embeddedness means that the boundaries of a CAS are blurred because each CAS is embedded within other CASs and a wider context. For example, a public hospital can be viewed as one CAS and it is embedded in a regional healthcare system, which is embedded in a national healthcare system, which is embedded in large industrial, societal and political systems.

- Non-linearity means that, in a CAS, the magnitude of system input and agent interactions are not linearly related to the magnitude of changes; a small change can bring large effects, while adversely, large changes may only have minimal impacts.

- Co-evolution is more likely the response to non-linearity and embeddedness as it indicates that components of one system and all related systems evolve together because of complex interdependencies and ongoing interactions among elements. The evolution of each of these systems flexibly reacts to both formal and informal changes in the context to ensure optimal functioning and survival. As a result, in CASs, change represents the process of co-evolution with other related systems while relationships are not unidirectional and isolated but connected and reciprocal.

- Self-organization describes the rules of change in a CAS as it indicates that autonomous agents in a systematic pattern respond to their environment using internalized rule sets and interact with each other to create outcomes. Based on this regularity, it can be assumed that innovation, order, and progress in a CAS can emerge naturally and do not need to be imposed centrally or from outside.

- Attractor patterns are used to represent the core properties of a system which always tends to return regardless of change (Theodoridis, 2009). Attractor patterns usually act as magnetic forces that draw CAS towards given trajectories (Pascale, Milleman, \& Gioja, 1999) and provide a reasonable explanation for those unpredictable changes that may not be logical but non-ignorable.

As P. E. Plsek and Greenhalgh (2001) outlined, the application of attractor patterns in complex theory provides a comparatively simple understanding of behaviour that is believed as extremely complex in the first place. Relative examples include psychotherapy process as clients are more likely to follow a counsellor's direction and accept his/her advice if the consulting conversation is framed in ways that enhance their core sense of integrity, autonomy, and ideals. Based on the above-mentioned expression, it is argued that the healthcare organization is one of the typical CASs as all its components adhere to the principles of complexity theory and are closely connected and interacted with each other. Any observable outcomes resulted from the system change are more than the sum of the parts. Therefore, the complexity theory can be applied in coping with healthcare social work of senior people, which is modelled as the CAS. In the healthcare social work involving senior people, the behaviour of any specific practice will be influenced by both internal and external factors because of the existence of intersectional connections within and between systems. Thereby, understanding the key features of the proposed healthcare social work system can implicate changes in systems and will largely help practitioners build a deep understanding about the complex cases on hand and adjust their practice with clients.

\section{INTEGRATING THE COMPLEXITY THEORY WITH CON- TEMPORARY HOSPITAL SOCIAL WORK PRACTICE}

Successful social work intervention requires social workers working together with their clients to fully identify 
the strengths and weaknesses that prompt or constrain changes. However, because of the complex nature of seniors' health conditions, it is quite hard for social workers to arise up a common effective working strategy, especially in CASs like the public hospital. The main aim of this study is to integrate the complexity theory with hospital social work with senior people. As per description and analysis, the work of integration obeys the principle of characteristics decomposition of the CAS in the following process: (1) Specify the core problems of the proposed object; (2) Manifest the elements of the system and their interrelatedness; (3) Clarify the specified impact factors of the study. As a theoretical approach, complexity theory is capable of deconstructing deconstruct complex behaviour which arises from the interaction between components of large collections in many disciplines. Through emphasising the importance of understanding self-organising patterns, attractor patterns and co-evolution process of one CAS, social workers will be able to rationally analyse the constraint conditions of the complex cases and adopt the most efficient interventions accordingly.

Firstly, in terms of redefining roles of hospital social workers and promoting their value in a multi-disciplinary team, especially when working with senior patients, the complexity theory can be adopted. This is because the understanding of complexity theory can provide health workers with a critical perspective about the positivist assumptions which often performs an ideological role in promoting "technocratic expertise and managerial authority" (Bhaskar, 2009). This assumption often underlies models for interprofessional collaboration, and a critical view about this privilege can adversely help professionals view interprofessional collaboration in a more objective perspective and redefine their specific duty in a multi-professional team. For example, many interprofessional allied health teams in the hospital are leaded by a nurse manager who may value medical treatment over other services. If complexity theory is properly understood by other team members, they will understand any success related to a complex case could not be achieved by effort in a single aspect. Thereby, when dealing with complex cases, this medical dominated authority is more likely to be challenged and further cooperation within the team will be promoted. As a result, better teamwork results and high service quality can be achieved.

Additionally, complex theory can provide a conceptual and theoretical analysis tool for social workers to have a better understanding of individuals' behaviour and contextual factors, and further optimize their intervention strategies. As an emerging discipline that challenges the dominant scien- tific approach called "reductionism", the complexity theory encourages allied health workers to abandon traditional linear models and accept unpredictability, autonomy and creativity of their work (Kernick, 2006; P. E. Plsek \& Greenhalgh, 2001). That is to say when working with senior patients, social workers should first view the whole cases in the broad social, political, economic and cultural contexts, and then analyse the limited or promoted factors for changes with the consideration of the interconnection and interaction of different components. For instance, the diagnosis of diabetes may change the dietary structure of one aged adult, which could further impact patients' social circle as he may need to reduce the frequency of dining out with his friends. After examining the possible changing patterns, social work interventions also should be considered in patients' contexts in order to reach the best service result. Just as noted by Kernick (2002), complexity theory can help social workers in hospitals reflect the multi-layered reality in the healthcare system by explaining the interconnected relationship between macro-structures (organizational) and micro-level behaviour (individual) of the system.

Furthermore, it is argued that the integration of complex theory with other social work theories will help social work practitioners rebuild their framework for practice and prompt them to change their working strategies with older people. It is worth noting that this theory not only provides an analytical tool and a paradigm for problemsolving but also works as a practical and instructive theory that complements the existing approaches by alerting social workers about the importance of matching complex problems to the applied context and environment. According to Kernick (2006), the insights provided by complexity theory resonate with the way in which social work practitioners view the world and offer a framework within which social workers can articulate and explore their intuitive insights. Through integrating complexity theory with social work practice framework, social workers will be able to combine quantitative and qualitative data that reflect the reality of complex cases without privileging either mode, which helps them more effectively and reasonably recognize those inevitable and necessary factors that facilitate clients' social engagements. For social workers in the hospital, this means social workers will more readily develop an appreciation of how their practice feeds into the activity of the network and formulate specific intervention on a case-by-case basis when they work with elder patients in complex situations. 


\section{CONCLUSION}

In this study, a reasonable approach for comprehending and analysis complexity is described, specifically in the field of hospital social work practice with senior people. The paper uses the degree of interrelations and difficulties of predictable as the indicator of the complexity, and views the system of hospital social work with senior people as a CAS since the individual components and holistic system comply the relevant key features. By considering the CAS as the smaller functionally components, the interrelations are studied based on the rational and practical considerations. Some practical points are promoted for hospital social workers to integrate complexity theory with their real practice and ease their physical and emotional burden. The functional decomposition and the holistic consideration of macro, meso and micro contexts of senior patients are beneficial for hospital social workers to strengthen the synthetic understanding of work context and to enable a reasonable view of refining social work practice at the individual and team practice levels.

\section{REFERENCES}

Albert, C., \& Davia, M. A. (2011). Education is a key determinant of health in Europe: A comparative analysis of 11 countries. Health Promotion International, 26(2), 163-170. doi:https://doi.org/10.1093/heapro/daq059

Australian Department of Human Services. (2017). Medicare Benefits Schedule (MBS) : Items by patient demographics report. Retrieved from https://bit.1y/2Qb85CU

Australian Institute of Health and Welfare. (2018). Older australia at a glance. Retrieved from https://bit. Iy/2QuF05N

Bhaskar, R. (2009). Scientific realism and human emancipation. London, UK: Routledge.

Campbell, N. C., Murray, E., Darbyshire, J., Emery, J., Farmer, A., Griffiths, F., ... Ann, L. (2007). Designing and evaluating complex interventions to improve health care. Bmj, 334(7591), 455-459. doi:https://doi.org/10.1136/bmj.39108 .379965.be

Craig, S. L., Bejan, R., \& Muskat, B. (2013). Making the invisible visible: Are health social workers addressing the social determinants of health? Social Work in Health Care, 52(4), 311-331. doi:https://doi.org/10.1080/00981389.2013 .764379

Dong, L., \& Keshavjee, K. (2016). Why is information governance important for electronic healthcare systems? A Canadian experience. Journal of Advances in Humanities and Social Sciences, 2(5), 250-260. doi:https://doi.org/10.20474/ jahss-2.5.1

Fish, S., \& Hardy, M. (2015). Complex issues, complex solutions: Applying complexity theory in social work practice. Nordic Social Work Research, 5(1), 98-114. doi:https://doi.org/10.1080/2156857x.2015.1065902

Hautsalo, K., Rantanen, A., \& Astedt-Kurki, P. (2013). Family functioning, health and social support assessed by aged home care clients and their family members. Journal of Clinical Nursing, 22(19-20), 2953-2963. doi:https://doi.org/10 $.1111 / \mathrm{j} .1365-2702.2012 .04335 . x$

Health Care. (2010). Patient protection and affordable care act. Retrieved from https://bit. Iy/2UdSPGB

Hudson, C. G. (2000). At the edge of chaos: A new paradigm for social work? Journal of Social Work Education, 36(2), 215-230. doi:https://doi.org/10.1080/10437797.2000.10779003

Israel, N., \& Wolf-Branigin, M. (2011). Nonlinearity in social service evaluation: A primer on agent-based modeling. Social Work Research, 35(1), 20-24. doi:https://doi.org/10.1093/swr/35.1.20

Kannampallil, T. G., Schauer, G. F., Cohen, T., \& Patel, V. L. (2011). Considering complexity in healthcare systems. Journal of Biomedical Informatics, 44(6), 943-947. doi:https://doi.org/10.1016/j.jbi.2011.06.006

Kernick, D. (2002). Complexity and healthcare organisation. In Complexity and healthcare: An introduction. Abingdon, UK: Radcliffe Medical Press.

Kernick, D. (2006). Wanted-new methodologies for health service research: Is complexity theory the answer? Family Practice, 23(3), 385-390. doi:https://doi.org/10.1093/fampra/cml011

Litaker, D., Tomolo, A., Liberatore, V., Stange, K. C., \& Aron, D. (2006). Using complexity theory to build interventions that improve health care delivery in primary care. Journal of General Internal Medicine, 21(2), 30-34. doi:https://doi.org/ 10.1007/s11606-006-0272-z

Long, K. M., McDermott, F., \& Meadows, G. N. (2018). Being pragmatic about healthcare complexity: Our experiences applying complexity theory and pragmatism to health services research. BMC Medicine, 16(1), 94-110. doi:https://doi.org/ 10.1186/s12916-018-1087-6 
Lymbery, M. (2006). United we stand? Partnership working in health and social care and the role of social work in services for older people. British Journal of Social Work, 36(7), 1119-1134. doi:https://doi.org/10.1093/bjsw/bch348

McAlinden, F., McDermott, F., \& Morris, J. (2013). Complex patients: Social workers' perceptions of complexity in health and rehabilitation services. Social Work in Health Care, 52(10), 899-912. doi:https://doi.org/10.1080/00981389.2013 .834032

McNamara, M., Bohle, P., \& Quinlan, M. (2011). Precarious employment, working hours, work-life conflict and health in hotel work. Applied Ergonomics, 42(2), 225-232. doi:https://doi.org/10.1016/j.apergo.2010.06.013

Miller, W. L., Crabtree, B. F., McDaniel, R., \& Stange, K. C. (1998). Understanding change in primary care practice using complexity theory. Journal of Family Practice, 46(5), 369-376.

Newhouse, R. P., \& Spring, B. (2010). Interdisciplinary evidence-based practice: Moving from silos to synergy. Nursing Outlook, 58(6), 309-317. doi:https://doi.org/10.1016/j.outlook.2010.09.001

Omorogbe, C. E. (2018). Socio-economic factors influencing in-patient satisfaction with health care at the University of Benin Teaching Hospital (UBTH), Benin city, Nigeria. International Journal of Nursing, Midwife and Health Related Cases, 4(4), 63-80.

Pascale, R. T., Milleman, M., \& Gioja, L. (1999). Surfing the edge of chaos. Creative Management and Development, 4(5), 45-50.

Plsek, P. (2003). Complexity and the adoption of innovation in health care (Technical report). National Institute for Healthcare Management Foundation and National Committee for Quality in Health Care, Washington, DC, WA.

Plsek, P. E., \& Greenhalgh, T. (2001). The challenge of complexity in health care. Bmj, 323(7313), 625-628. doi:https:// doi.org/10.1136/bmj.323.7313.625

Redfern, H., Burton, J., Lonne, B., \& Seiffert, H. (2016). Social work and complex care systems: The case of people hospitalised with a disability. Australian Social Work, 69(1), 27-38. doi:https://doi.org/10.1080/0312407x.2015.1035295

Saito, E., Sagawa, Y., \& Kanagawa, K. (2005). Social support as a predictor of health status among older adults living alone in Japan. Nursing \& Health Sciences, 7(1), 29-36. doi:https://doi.org/10.1111/j.1442-2018.2005.00220.x

Theodoridis, C. (2009). Complexity in retail organisations: An empirically informed discussion with particular reference to retail location strategy. Retrieved from https://bit.ly/3aRpdFN

Tokuda, K. (2016). Individuals' compassion and organizational inclusiveness: Case studies of Japanese BCTA companies fighting for global health. Journal of Advanced Research in Social Sciences and Humanities, 1(1), 47-51. doi:https:// doi.org/10.26500/jarssh-01-2016-0106

Tuffin, R. (2016). Implications of complexity theory for clinical practice and healthcare organization. Bja Education, 16(10), 349-352. doi:https://doi.org/10.1093/bjaed/mkw013

Warren, K., Franklin, C., \& Streeter, C. L. (1998). New directions in systems theory: Chaos and complexity. Social Work, 43(4), 357-372. doi:https://doi.org/10.1093/sw/43.4.357

Warren, K., Hawkins, R. C., I, \& Sprott, J. C. (2003). Substance abuse as a dynamical disease: Evidence and clinical implications of nonlinearity in a time series of daily alcohol consumption. Addictive Behaviors, 28(2), 369-374. doi:https://doi.org/ 10.1016/s0306-4603(01)00234-9

Woehle, R. (2007). Complexity theory, nonlinear dynamics, and change: Augmenting systems theory. Advances in Social Work, 8(1), 141-151. doi:https://doi.org/10.18060/137

Yi, K. H., \& Chia, N. L. (2018). Research on the evaluation indexes of walking friendly environment in healthy communities from the perspective of mass transit oriented Development -Taipei MRT as an example. International Journal of Humanities, Arts and Social Sciences, 4(1), 1-14. doi:https://doi.org/10.20469/ijhss.4.10001-1 